PROCEEDINGS OF THE

AMERICAN MATHEMATICAL SOCIETY

Volume 133, Number 9, Pages 2609-2611

S 0002-9939(05)08089-5

Article electronically published on April 25, 2005

\title{
STRONG CONVERSE INEQUALITY FOR POISSON SUMS
}

\author{
F. DAI AND Z. DITZIAN
}

(Communicated by Andreas Seeger)

\begin{abstract}
The rate of convergence of Poisson sums and their combinations are shown to be equivalent to appropriate $K$-functionals.
\end{abstract}

For a function $f$ that has the expansion

$$
f(x) \sim \sum_{k=0}^{\infty} P_{k}(f)
$$

the Poisson sum is given by

$$
A_{r} f=\sum_{k=0}^{\infty} r^{k} P_{k}(f), \quad 0<r<1 .
$$

For $A_{r} f$ to be written as a semi-group, we set $r=e^{-t}$ and obtain

$$
T(t) f=\sum_{k=0}^{\infty} e^{-k t} P_{k}(f) .
$$

For the purpose of this paper, $H_{k}$ are eigenspaces of a given self-adjoint operator $P(D)$ with eigenvalues $\lambda_{k}$ such that $\lambda_{k}$ are either all positive or all negative, and $(-1)^{j} \lambda_{k}=\left|\lambda_{k}\right|<\left|\lambda_{k+1}\right|$. Moreover, $\lambda_{k}$ is a polynomial in $k$. We deal with a Banach space of functions $B$ such that $H_{k} \subset B, H_{k} \subset B^{*}$, span $\bigcup_{k=0}^{\infty} H_{k}$ is dense in $B$ and $P_{k}(f)$ is the projection of $f$ onto $H_{k}$ (see [Ch-Di]).

We further assume that the Cesàro summability of order $\ell$, for some $\ell$, is a contraction on $B$, that is,

$$
\left\|C_{n}^{\ell} f\right\|_{B} \leq\|f\|_{B} .
$$

We note that if $C_{n}^{\ell} f$ is a contraction, so is $C_{n}^{m} f$ for $m>\ell$. We also note that in all cases known to us $C_{n}^{\ell} f$ is a contraction when it is a positive operator. If $C_{n}^{\ell}$ is a contraction on $B$, so is the Poisson sum $A_{r} f$, which can be written (see [Ha, p. 108] or simply summation by parts) as

$$
A_{r} f=(1-r)^{1+\ell} \sum_{k=0}^{\infty} A_{k}^{\ell} r^{k} \sigma_{k}^{\ell}(f), \quad A_{k}^{\ell}=\left(\begin{array}{c}
k+\ell \\
k
\end{array}\right),
$$

and clearly $T(t) f$ is also a contraction. (Positivity is also inherited.)

Received by the editors January 27, 2004.

2000 Mathematics Subject Classification. Primary 41A17, 41A63.

This work was supported by NSERC grant of Canada A4816.

(C)2005 American Mathematical Society Reverts to public domain 28 years from publication 
The infinitesimal generator of $T(t) f$ is formally given by

$$
\mathcal{A} f \sim \sum_{k=1}^{\infty}-k P_{k}(f)
$$

and the domain of $\mathcal{A}, D(\mathcal{A})$ in $B$ is all $f$ in $B$ such that $\mathcal{A} f \in B$ where $\mathcal{A} f$ is given by (6).

We now have the following result.

Theorem 1. If for a given space $B, C_{n}^{\ell} f$ satisfies (4), then $\mathcal{A T}(t) f \in B$ for all $t>0$ and

$$
t\|\mathcal{A} T(t) f\|_{B} \leq 2(\ell+2)(\ell+1)\|f\|_{B} ;
$$

that is, $T(t)$ is a holomorphic semigroup of contractions.

Proof. Clearly, $\mathcal{A} C_{n}^{\ell}(f)$ is in $B$. A simple calculation yields

$$
\mathcal{A} C_{n}^{\ell}(f)=(n+\ell+1)\left(C_{n}^{\ell+1}(f)-C_{n}^{\ell}(f)\right),
$$

and hence

$$
\begin{aligned}
\mathcal{A} T(t) f & =\left(1-e^{-t}\right)^{\ell+1} \sum_{k=0}^{\infty} A_{k}^{\ell} e^{-t k} \mathcal{A} C_{k}^{\ell}(f) \\
& =\left(1-e^{-t}\right)^{\ell+1} \sum_{k=1}^{\infty} A_{k}^{\ell} e^{-t k}(k+\ell+1)\left(C_{n}^{\ell+1}(f)-C_{n}^{\ell}(f)\right) .
\end{aligned}
$$

Therefore,

$$
\begin{aligned}
\|\mathcal{A} T(t) f\|_{B} & \leq\left(1-e^{-t}\right)^{\ell+1} \sum_{k=1}^{\infty} A_{k}^{\ell} e^{-t k}(k+\ell+1)\left(\left\|C_{n}^{\ell+1}(f)\right\|_{B}+\left\|C_{n}^{\ell}(f)\right\|_{B}\right) \\
& \leq 2\|f\|_{B}\left(1-e^{-t}\right)^{\ell+1} \sum_{k=1}^{\infty} A_{k}^{\ell} e^{-t k} k(\ell+2) \\
& \leq 2(\ell+2)\|f\|_{B}\left(1-e^{-t}\right)^{\ell+1}\left(-\frac{d}{d t} \frac{1}{\left(1-e^{-t}\right)^{\ell+1}}\right) \\
& \leq 2(\ell+2)\|f\|_{B}(\ell+1) \frac{e^{-t}}{\left(1-e^{-t}\right)^{\ell+2}}\left(1-e^{-t}\right)^{\ell+1} \\
& \leq 2(\ell+2)(\ell+1) \frac{e^{-t}}{1-e^{-t}}\|f\|_{B},
\end{aligned}
$$

and as $\frac{t e^{-t}}{1-e^{-t}} \leq 1$ for $t>0,(7)$ follows.

We can now follow Di-Iv Section 5, Theorem 5.1] and deduce the following result as an immediate corollary.

Theorem 2. Let $T(t) f$ and $A_{r} f$ be given by (3) and (2) respectively, let $\mathcal{A}$ be given by (6) and suppose (4) is satisfied for some integer $\ell$. Then

$$
\left\|(T(t)-I)^{m} f\right\|_{B} \approx \inf _{g \in D\left(\mathcal{A}^{m}\right)}\left(\|f-g\|_{B}+t^{m}\left\|\mathcal{A}^{m} g\right\|_{B}\right), \quad t>0,
$$


and

(9) $\left\|\sum_{k=0}^{m-1}\left(\begin{array}{c}m \\ k\end{array}\right)(-1)^{k} A_{r m-k} f+(-1)^{m} f\right\|_{B}$

$$
\approx \inf _{g \in D\left(\mathcal{A}^{m}\right)}\left(\|f-g\|_{B}+(1-r)^{m}\left\|\mathcal{A}^{m} g\right\|_{B}\right), \quad 0<r<1,
$$

where $D\left(\mathcal{A}^{m}\right)$ is the domain of $\mathcal{A}^{m}$ in $B$.

Remark 3. If $\lambda(k)$ is a polynomial of degree $b$ in $k$, a simple calculation or Lemma 3.2

of [Da shows that domain $\mathcal{A}$ is the same as the domain of $\left((-1)^{j} P(D)\right)^{1 / b}$ and $\left\|\mathcal{A}^{m} g\right\|_{B} \approx\left\|(-1)^{j} P(D)^{m / b} g\right\|_{B}$ with

$$
\left((-1)^{j} P(D)\right)^{\alpha} g \sim \sum_{k=0}^{\infty} \lambda(k)^{\alpha} P_{k}(g)
$$

as defined in [Di].

\section{REFERENCES}

[Ch-Di] W. Chen and Z. Ditzian, Best approximation and K-functionals, Acta Math. Hungar. 75 (1997), 165-208. MR1440498 (98h:41013)

[Da] F. Dai, Some equivalence theorems with K-functionals, Jour. of Approx. Theory 121 (2003), 143-157. MR.1963000 (2003m:42013)

[Di] Z. Ditzian, Fractional derivatives and best approximation, Acta Math. Hungar. 81 (4) (1998), 323-348. MR1662219 (2000e:41046)

[Di-Iv] Z. Ditzian and K. Ivanov, Strong converse inequalities, Jour. d'Analyse Math. 61 (1993), 61-111. MR 1253439 (94m:41038)

[Ha] G.H. Hardy, Divergent Series, Oxford Univ. Press, 1948. MR0030620 (11:25a)

Department of Mathematical and Statistical Sciences, University of Alberta, Edmonton, Alberta, Canada T6G 2G1

Department of Mathematical and Statistical Sciences, University of Alberta, Edmonton, Alberta, Canada T6G 2G1 